\title{
Lanthanide-Assisted Deposition of Strongly Electro-optic PZT Thin Films on Silicon: Toward Integrated Active Nanophotonic Devices
}

\author{
J. P. George, ${ }^{*, \dagger, \ddagger, \|}$ P. F. Smet, ${ }^{\S, \|}$ J. Botterman, $^{\S, \|}$ V. Bliznuk, ${ }^{\perp}$ W. Woestenborghs, ${ }^{\dagger, \|}$ D. Van Thourhout, ${ }^{\ddagger, \|}$ \\ K. Neyts, ${ }^{\dagger, \|}$ and J. Beeckman ${ }^{\dagger, \|}$
}

${ }^{\dagger}$ Department of Electronics and Information Systems, Ghent University, Sint-Pietersnieuwstraat 41, 9000 Gent, Belgium

${ }^{\ddagger}$ Department of Information Technology, Ghent University, Sint-Pietersnieuwstraat 41, 9000 Gent, Belgium

${ }^{\S}$ LumiLab, Department of Solid State Sciences, Ghent University, Krijgslaan 281-S1, 9000 Gent, Belgium

"Center for Nano- and Biophotonics (NB-Photonics), Ghent University, 9000 Gent, Belgium

${ }^{\perp}$ Department of Materials Science and Engineering, Ghent University, Technologiepark 903, B-9052 Zwijnaarde, Belgium

Supporting Information

ABSTRACT: The electro-optical properties of lead zirconate titanate (PZT) thin films depend strongly on the quality and crystallographic orientation of the thin films. We demonstrate a novel method to grow highly textured PZT thin films on silicon using the chemical solution deposition (CSD) process. We report the use of ultrathin $(5-15 \mathrm{~nm}$ ) lanthanide (La, Pr, Nd, Sm) based intermediate layers for obtaining preferentially (100) oriented PZT thin films. $\mathrm{X}$-ray diffraction measurements indicate preferentially oriented intermediate $\mathrm{Ln}_{2} \mathrm{O}_{2} \mathrm{CO}_{3}$ layers providing an excellent lattice match with the PZT thin films grown on top. The XRD and scanning electron microscopy measurements reveal that the annealed layers are dense, uniform, crack-free and highly oriented $(>99.8 \%)$ without apparent defects or secondary phases. The EDX and HRTEM characterization confirm that the template layers act as an efficient diffusion barrier and form a sharp interface between the substrate and
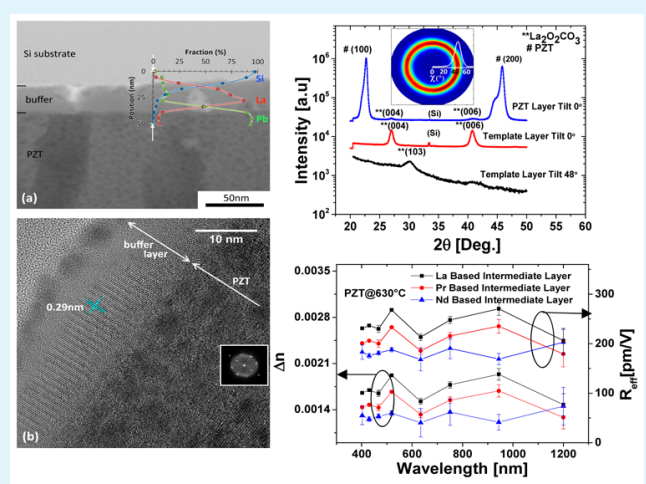
the PZT. The electrical measurements indicate a dielectric constant of $\sim 650$, low dielectric loss of $\sim 0.02$, coercive field of $70 \mathrm{kV} / \mathrm{cm}$, remnant polarization of $25 \mu \mathrm{C} / \mathrm{cm}^{2}$, and large breakdown electric field of $1000 \mathrm{kV} / \mathrm{cm}$. Finally, the effective electro-optic coefficients of the films are estimated with a spectroscopic ellipsometer measurement, considering the electric field induced variations in the phase reflectance ratio. The electro-optic measurements reveal excellent linear effective pockels coefficients of 110 to $240 \mathrm{pm} / \mathrm{V}$, which makes the CSD deposited PZT thin film an ideal candidate for Si-based active integrated nanophotonic devices.

KEYWORDS: chemical solution deposition, electro-optic effect, ellipsometry, dielectric, lead zirconate titanate, modulator, pockels coefficient

\section{INTRODUCTION}

The deposition of high quality ferroelectric thin films has been studied extensively over the past few decades, because of the excellent piezo-electric, ${ }^{1}$ pyro-electric, ${ }^{2}$ ferro-electric, ${ }^{3}$ and electro-optic ${ }^{4}$ properties. The presence of functional properties in many ferroelectric materials has motivated the heterogeneous integration of crystalline thin films directly on silicon for the realization of a variety of novel devices. Among the known ferroelectric thin film oxides, lead zirconate titanate (PZT) exhibits a large dielectric constant, piezo-electric coefficient ${ }^{5,6}$ and electro-optic coefficient, ${ }^{4}$ which already lead to applications in ferro-electric thin film capacitors, ${ }^{7}$ piezo-electric actuators, ${ }^{8}$ and electro-optic modulators. ${ }^{9,10}$ Recent advancements in the thin film technology show nanograined PZT film ${ }^{11}$ with excellent ferroelectric properties. ${ }^{11,12}$ Novel devices with PZT nanofiber have been demonstrated for mechanical energy harvesting. ${ }^{13}$ Other interesting properties of the films such as the electron emission characteristics are also explored with nanograined PZT structures, and a low turn on electric field is reported. ${ }^{14}$ Although the ferroelectric thin films are explored mostly for electronic applications, the ever increasing demand for higher bandwidth meanwhile putting constraints on power consumption, has resulted in an increasing interest toward the use of the films in the optical domain. To realize optical data transmission, for example, for chip-to-chip interconnects, integrated optical devices which are compatible with the electrical systems are indispensable. The well-established ferroelectric oxide based technologies propelled the development of high-speed electro-optic devices on different material platforms, however the silicon based photonics technologies have not explored the strong linear electro-optic (EO) properties of PZT thin films. ${ }^{15}$ The current state of the art

Received: February 26, 2015

Accepted: June 4, 2015

Published: June 4, 2015 
high-speed integrated optical devices are based on $\mathrm{LiNbO}_{3}$ single crystals. ${ }^{16}$ However, replacing them with ferroelectric thin film oxides such as PZT, along with the compact silicon photonic platform, could be an alternative to realize smaller, functional and power efficient devices. ${ }^{10,17-19}$

A variety of methods has been used to develop ferroelectric thin films: chemical solution deposition (CSD) ${ }^{20}$ RF magnetron sputtering, ${ }^{21}$ metal organic chemical vapor deposition $(\mathrm{MOCVD})^{22}$ and pulsed laser deposition (PLD). ${ }^{23}$ However, the direct deposition of PZT on silicon still remains a challenge. It is reported that there is interdiffusion between $\mathrm{Si}$ and PZT thin film at a high annealing temperature, which makes it hard to form the perovskite PZT phase. ${ }^{24}$ Different methods have been proposed and demonstrated to use either a seed or a barrier layer to promote thin film growth. ${ }^{25}$ The nucleation of the PZT thin films strongly depends on the underlying substrate and the interface properties. ${ }^{26}$ The dielectric, piezoelectric and electro-optical properties of the thin films depend strongly on the crystal orientation. It has been reported that strongly $c$-axis-oriented PZT thin films can be grown on either single-crystalline oxide substrates or by using a preferentially oriented thick $(>100 \mathrm{~nm})$ conductive or dielectric intermediate buffer layer. ${ }^{27}$ Metallic intermediate layers are preferred for electrical devices, but often such layers cannot be used for electro-optical devices because the optical absorption is too high. A thick intermediate layer is not necessarily a drawback for certain applications of PZT, but for other applications such as electro optical devices the performance is severely limited by the thickness of this layer. However, recent works show advancements toward micro and nano scale EO devices on the silicon platform based on complex and costly layer bonding approaches ${ }^{28}$ and with the more sophisticated epitaxially grown ferroelectric oxides. ${ }^{10}$

In this work, we demonstrate a novel technique to realize textured PZT thin films on a variety of substrates such as bare silicon, glass (corning glass type CB90IN (Delta Technologies)), glass/ITO, $\mathrm{Si} / \mathrm{Al}_{2} \mathrm{O}_{3}, \mathrm{Si} / \mathrm{SiO}_{2}$, and $\mathrm{Si} / \mathrm{Si}_{3} \mathrm{~N}_{4}$, with a conventional 2-methoxy ethanol based CSD technique. ${ }^{27}$ Ultrathin dielectric intermediate layers of 5 to $10 \mathrm{~nm}$ have been used as an efficient intermediate layer to promote the thin film growth. The previous reported methods that use thin template layers are either based on epitaxially grown $\mathrm{SrTiO}_{3}{ }^{29}$ intermediate layers of $4-10 \mathrm{~nm}$ by molecular beam epitaxy (MBE) or on a thin intermediate thermally grown intermediate $\mathrm{SiO}_{2}$ layer with thickness ranging from 5 to 10 nm. ${ }^{24}$ The $\mathrm{SrTiO}_{3}$ template layer results in epitaxial PZT thin films, but this growth technique is limited to crystalline substrates $(\mathrm{Si})$, whereas in the latter technique the phase purity of the films critically depends on the $\mathrm{SiO}_{2}$ thickness. The possibility of chemical reaction at the $\mathrm{PZT} / \mathrm{SiO}_{2} / \mathrm{Si}$ interface at high temperature has also not been discussed there. In this work, the annealing temperature is significantly lower, while the film quality is quasi-independent of the type of substrate or the intermediate layer thickness.

\section{EXPERIMENTAL SECTION}

Solution Preparation. We use lanthanide-based thin films as intermediate layers for the PZT deposition. Nitrate salts of the lanthanides (La, Pr, Nd, Sm) are dissolved in propanol, and refluxed for $24 \mathrm{~h}$ at room temperature. The molarity of the solution is $0.02 \mathrm{M}$, which results in layers of about $10 \mathrm{~nm}$ thickness, upon spin coating and subsequent heat treatment. The PZT (52/48) precursor solution is prepared with a $20 \mathrm{~mol} \%$ excess of lead to compensate for lead loss during the crystallization heat treatments. We follow a 2-methoxy ethanol based chemical solution route, using acetyl acetone as a chelating agent. First, the lead acetate hydrate salt is dissolved in 2methoxy ethanol and vacuum distilled for $3 \mathrm{~h}$. Later, Ti-isopropoxide and $\mathrm{Zr}$-isoproxide solutions are mixed and chelated in stoichiometric proportion inside a glovebox, before it was mixed with the distilled lead acetate to get the final solution. The molarity of the final solution is $0.4 \mathrm{M}$.

Deposition Procedure. First, the intermediate layers are spincoated onto the substrate at $3000 \mathrm{rpm}$ for $40 \mathrm{~s}$, and heat treated on a hot plate at $200{ }^{\circ} \mathrm{C}$ for $2 \mathrm{~min}$. Subsequently, the layers are annealed at 400 to $500{ }^{\circ} \mathrm{C}$ in a tube furnace. Upon annealing the films undergo a sequence of endothermic weight loss reactions to initiate the intermediate phase transformation. ${ }^{30}$ As the molarity of the solution is increased to get a larger thickness for the intermediate layers, it is noticed that the nonuniformity of the spin-coated layers also increased. To avoid this problem, we have followed a multilayer spin-coating and annealing procedure. Each individual layer is spin coated and subsequently annealed in air, and this process is repeated over several cycles to achieve a desired thickness. Intermediate layers of 5- $80 \mathrm{~nm}$ have been prepared with this procedure. Second, the PZT precursor solution is spin-coated onto the intermediate layer, at $3500 \mathrm{rpm}$ for 40 s, followed by pyrolysis at $300{ }^{\circ} \mathrm{C}$ to burn out the organic components. Each spin coating and pyrolysis step results in a layer of about $50 \mathrm{~nm}$, so the process cycle is repeated 4 times to obtain a film of thickness $200 \mathrm{~nm}$, prior to the annealing process.

Subsequently, the amorphous PZT thin films are annealed in a tube furnace at 500 to $600{ }^{\circ} \mathrm{C}$, under flowing $\mathrm{O}_{2}$ gas, $10-20 \mathrm{~min}$, to allow crystallization into the perovskite PZT phase. A steady oxygen flow of $150 \mathrm{Liter} /$ hour is maintained throughout the annealing procedure. The ramping rates for heating and cooling of the specimen in the annealing system are 100 and $-50{ }^{\circ} \mathrm{C} / \mathrm{min}$, respectively. This process is repeated until a desired thickness of the PZT film is achieved.

X-ray Diffraction Measurements. The samples are first cleaned with acetone, isopropanol, and deionized water. The measurements are carried out with a D8 Discover diffractometer (Bruker technologies Ltd.,) with $\mathrm{CuK} \alpha$ radiation. The diffractograms are recorded for $2 \theta$ angles between $15^{\circ}$ and $64^{\circ}$, with step size $0.004^{\circ}$ and time step $1.2 \mathrm{~s}$.

Focused Ion Beam Etching/Scanning Electron Microscopy. The cross section images and the transmission electron microscope (TEM) slices of the specimens are prepared by a FEI Nova 600 Nanolab Dual-Beam Focused Ion Beam system (FIB) and associated scanning electron microscope (SEM). It allows simultaneous milling and imaging of the specimens. The SEM column is equipped with a high-performance field-emission gun electron source, whereas the FIB system has a gallium Liquid Metal Ion Source (LMIS).

(Scanning) Transmission Electron Microscopy. Transmission electron microscopy (TEM) analysis is performed using a JEM2200FS FEG-TEM (Jeol), operated at $200 \mathrm{keV}$, and equipped with an in-column omega filter to reduce chromatic aberration. Energy dispersive X-ray (EDX) spectroscopy was used to measure the chemical composition in scanning TEM mode (STEM).

Atomic Force Microscopy. The surface roughness of the PZT thin films are measured by Atomic Force Microscopy (AFM) analysis. The measurements are carried out with a Digital Instruments Dimension 3100 scanning probe microscope with a nanoscope IIIa controller in contact mode.

$C-V, C-F$, and $I-V$ Characteristics. Prior to the measurements, a top electrode is deposited with either chromium $(\mathrm{Cr})$ or indium tin oxide (ITO) (area $=3.14 \mathrm{~mm}^{2}$, thickness $50-100 \mathrm{~nm}$ ) by e-gun evaporation. The $C-V$ and $C-F$ (up to $1 \mathrm{MHz}$ ) characteristics are measured with a HP4192ALF impedance analyzer. The capacitance is measured for a small AC voltage (oscillation level $\sim 0.1 \mathrm{~V}$ ) which is superposed on a DC voltage offset. $I-V$ measurement are performed with a Keithley 236 source measure unit.

$P-E$ Hysteresis Measurements. A Sawyer-Tower circuit is used to measure the hysteresis loop in the polarization-electric field diagram of the PZT films. The measurements are carried out at frequencies in the range from $100 \mathrm{~Hz}$ to $1 \mathrm{kHz}$ with a sinusoidal acvoltage with an amplitude of $10 \mathrm{~V}$ peak-to-peak. 

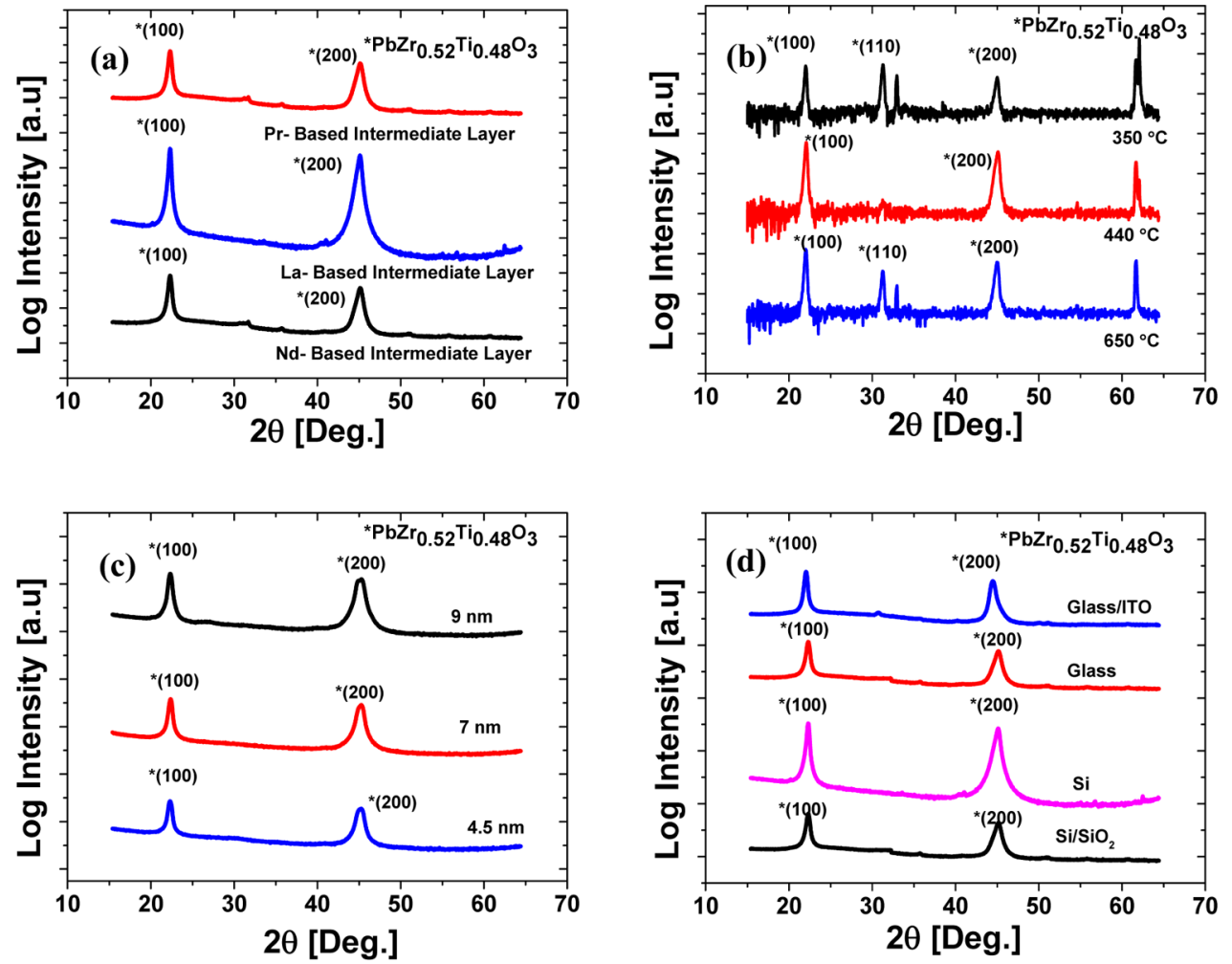

Figure 1. XRD patterns of PZT thin films annealed at $600{ }^{\circ} \mathrm{C}$. (a) PZT on intermediate layers of $\mathrm{La}$, $\mathrm{Pr}$, and Nd, which are heat treated at 500, 450, and $440{ }^{\circ} \mathrm{C}$, respectively. (b) On Nd-based intermediate layers of $10 \mathrm{~nm}$, which has been heat treated at different temperatures. (c) PZT on Si substrates coated with La-based buffer layers of different thickness (heat treated at $500{ }^{\circ} \mathrm{C}$ ). (d) PZT films on different substrates coated with Labased intermediate layers (thickness $\sim 10 \mathrm{~nm}$, heat treated at $500{ }^{\circ} \mathrm{C}$ ).

Spectroscopic Ellipsometer Measurements. Electro-optic characterizations of the PZT thin films are carried out with a spectroscopic ellipsometer (J. A. Woollam Co.). The ellipsometer is equipped with a Xe lamp source, with a rotating polarizer and analyzer.

\section{RESULTS AND DISCUSSION}

The thickness of the PZT film used for the XRD measurement is determined as $200 \pm 3 \mathrm{~nm}$ from spectroscopic ellipsometry measurements. Figure 1a represents a comparison between the X-ray diffractograms of the PZT thin films (annealed at 600 ${ }^{\circ} \mathrm{C}$ ) deposited on silicon substrates coated with intermediate layers of $\mathrm{La}, \mathrm{Pr}$, and $\mathrm{Nd}$, which are heat treated at 500, 450, and $440{ }^{\circ} \mathrm{C}$, respectively. The thickness of the intermediate layer is approximately $10 \mathrm{~nm}$ for this experiment. Regardless of the PZT processing conditions, such as pyrolysis temperature, pyrolysis time, or annealing temperature, the PZT films deposited onto these intermediate layers are crystallized into a pure perovskite phase, with no evidence of any intermediate secondary phase formation. The diffractograms show strong diffraction peaks along the (100) and (200) crystallographic orientations at $2 \theta=22^{\circ}$, with either tetragonal or rhombohedral structure. Figure $1 \mathrm{~b}$ represents the XRD patterns of the PZT films (annealed at $600{ }^{\circ} \mathrm{C}$ ) deposited on Nd-based intermediate layer, which are heat treated at different temperatures. It is evident that the layers that are heat treated at 350 and $650{ }^{\circ} \mathrm{C}$ show a mixed (100) and (110) orientation, whereas the intermediate layer heat treated at $440{ }^{\circ} \mathrm{C}$ results in a strong (100) preferential orientation. The heat treatment of the intermediate layer influences the nucleation mechanism, which results in different diffraction peaks in the XRD spectrum. It has been reported that heat treatment initiates the transformation of the nitrate film into different crystalline states of $\mathrm{NdO}_{0.5} \mathrm{NO}_{2}, \mathrm{NdONO}_{3}$, and $\mathrm{Nd}_{2} \mathrm{O}_{3}$ at 350, 440, and $650{ }^{\circ} \mathrm{C}$, respectively. Even though many crystalline phases exist on the thermal decomposition of $\mathrm{Nd}\left(\mathrm{NO}_{3}\right)_{3} \cdot 6 \mathrm{H}_{2} \mathrm{O},{ }^{31}$ we found that the intermediate phase obtained after a heat treatment around $440{ }^{\circ} \mathrm{C}$ is the only one resulting in oriented thin film growth. Furthermore, during the heat treatment $\mathrm{LnO}_{2} \mathrm{CO}_{3}$ is formed rather than the mixed $\operatorname{Ln}(\mathrm{O}, \mathrm{N})$ compounds, as will be motivated below, ${ }^{32,33,31}$. Figure $1 \mathrm{c}$ shows the X-ray diffractograms of the PZT thin films (annealed at $600{ }^{\circ} \mathrm{C}$ ) deposited on $\mathrm{Si}$ substrates coated with a La-based intermediate layer (heat treated at $500{ }^{\circ} \mathrm{C}$ ) of different thicknesses. When the intermediate layer thickness is only $3 \mathrm{~nm}$, the intermediate layers are not efficient to prevent the interdiffusion of $\mathrm{Si}$ and PZT, and only the pyrochlore PZT phase is observed. A similar result has been reported before with $\mathrm{PZT}$ film on ultrathin intermediate $\mathrm{SiO}_{2}$ of thickness $<4 \mathrm{~nm}^{24}$ However, as the intermediate layer thickness increases above $5 \mathrm{~nm}$, well-defined diffraction peaks corresponding to the stoichiometric PZT are observed along the (100) and (200) crystallographic directions. The same experiments are repeated on different substrates including $\mathrm{Si}$, glass, glass/ITO, $\mathrm{Si} / \mathrm{Al}_{2} \mathrm{O}_{3}, \mathrm{Si} / \mathrm{SiO}_{2}$, and $\mathrm{Si} / \mathrm{Si}_{3} \mathrm{~N}_{4}$. An apparent peak shift observed for the glass/ITO sample is due to the minor misalignment. All the PZT thin films show identical crystallographic orientation and similar properties, regardless of the substrate material. This indicates that the orientation of the thin film is not influenced by the substrate, and depends only on the nature of the intermediate layer. The minimum thickness of the intermediate layer required on bare silicon and glass substrates to avoid microcracks after high 
temperature annealing is estimated to be in the range of 9-15 $\mathrm{nm}$.

To analyze the mechanism of thin film growth, thicker Laintermediate layers $(\sim 60 \mathrm{~nm})$ are formed by repeating the spin coating and heat treatment procedure, without deposition of PZT on top. It is found that upon heat treatment at $500{ }^{\circ} \mathrm{C}$, the intermediate nitrate layer reacts with the $\mathrm{CO}_{2}$ present in the ambient air, resulting in the formation of tetragonal $\mathrm{La}_{2} \mathrm{O}_{2} \mathrm{CO}_{3}{ }^{31}(a=4.07 \AA, c=13.49 \AA)$. The XRD pattern shown in Figure 2 indicates that the intermediate layers have a

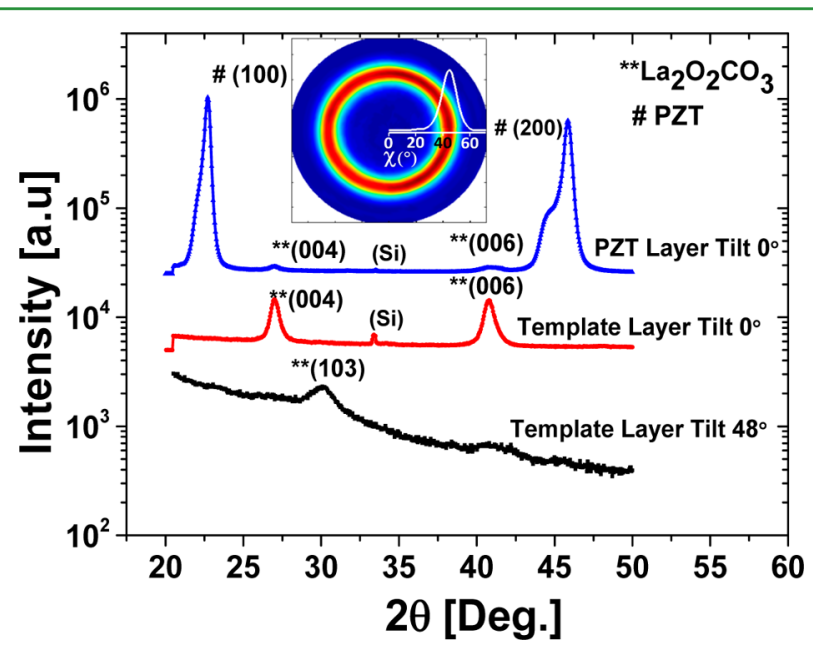

Figure 2. XRD pattern of the La-based intermediate layer, heat treated at $500{ }^{\circ} \mathrm{C}$ in air at a tilt angle of $0^{\circ}$ and $48^{\circ}$. Inset: X-ray pole figure of the PZT thin film at $2 \theta=30.95^{\circ}$ (showing the diffraction intensity of the (110) reflection as a function of tilt angle).

strong preferential growth with the $c$-axis perpendicular to the substrate, showing a good lattice match (mismatch in the $a$-axis parameters $<0.7 \%$ ) to PZT. The formation of $\mathrm{La}_{2} \mathrm{O}_{2} \mathrm{CO}_{3}$ is further confirmed upon recording the XRD at a tilt angle $\sim$ of $48^{\circ}$, bringing the (103) plane into diffraction (Figure 2).When annealing the intermediate layers in pure oxygen at low pressure, no distinct diffraction peaks are found, confirming that the formation of the carbonate compound plays a key role in the strong preferential orientation of the PZT thin film. The conclusions derived from X-ray diffraction are consistent with the microscopic behavior of the buffer layer, as studied with STEM and HRTEM (Figure 3b).

On the basis of the STEM image, it is clear that the $\mathrm{La}_{2} \mathrm{O}_{2} \mathrm{CO}_{3}$ layer acts as an efficient buffer layer between the $\mathrm{Si}$ substrate and the PZT layer. Also, the brightness variations in the PZT layer suggest the onset of the growth of columnar grains. A line scan by means of EDX was recorded to study the chemical composition of the buffer layer. The $\mathrm{La}_{2} \mathrm{O}_{2} \mathrm{CO}_{3}$ thin film indeed acts as an efficient buffer layer between the substrate and the PZT thin film (Figure 3a), since the compositional gradient for the representative elements $\mathrm{Si}, \mathrm{La}$, and $\mathrm{Pb}$ is steep, taking into account the typical spatial resolution of EDX in STEM. The HRTEM image (Figure 3b) confirms the crystalline nature of the buffer layer. In addition, the $c$-axis is found to be perpendicular to the interface between the PZT and buffer layer, in line with the texture analysis (Figure 2). Furthermore, the PZT layer is well crystallized, starting at the interface with the buffer layer. Again, the crystallographic orientation, as observed from the FFT power spectrum, is in line with the X-ray diffraction
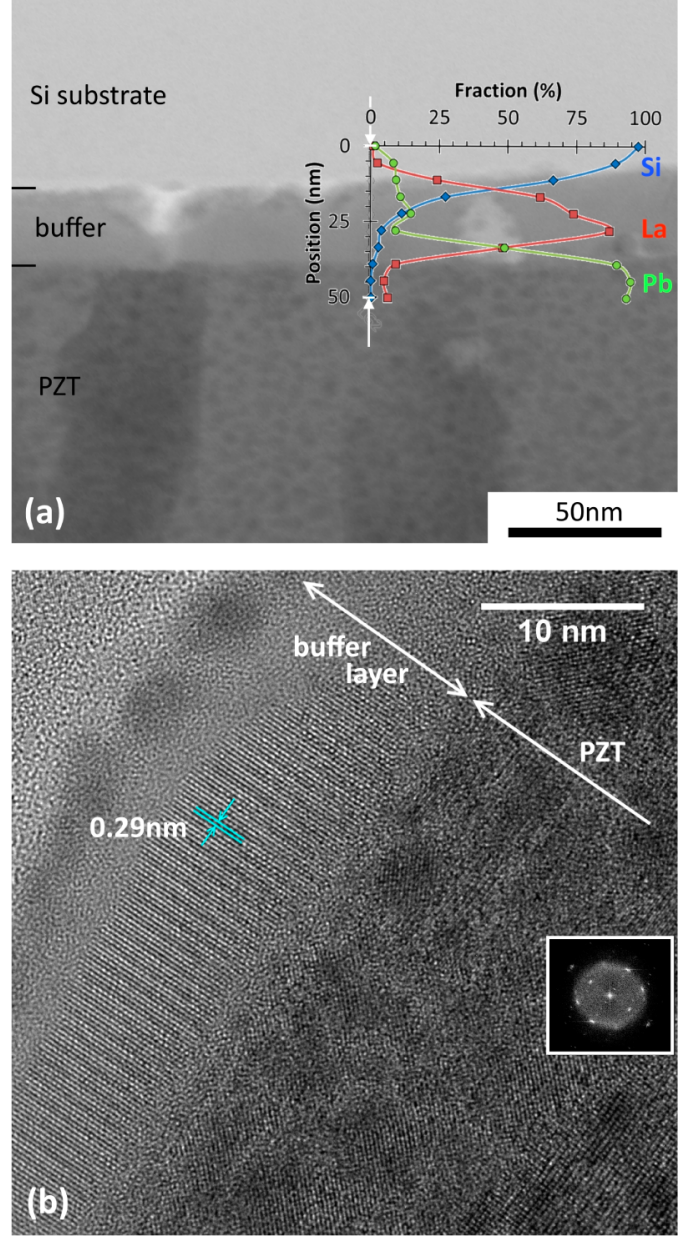

Figure 3. (a) STEM image of the cross section of the PZT thin film (annealed at $630{ }^{\circ} \mathrm{C}$ ) on top of the $\mathrm{La}_{2} \mathrm{O}_{2} \mathrm{CO}_{3}$ buffer layer (heat treated at $500{ }^{\circ} \mathrm{C}$ ). The overlay shows the relative EDX signal intensity measured along the line marked by the two white arrows. For these intensities, only the elements $\mathrm{La}, \mathrm{Pb}$ and $\mathrm{Si}$ were taken into account. (b) HRTEM image of the same cross-section, with indication of the (110) plane spacing in $\mathrm{La}_{2} \mathrm{O}_{2} \mathrm{CO}_{3}$. The inset shows the FFT power spectrum for the PZT layer.

measurements. The (110) planes of PZT are perpendicular to the interface and parallel to the incident electron beam. Consequently, there is epitaxial growth in the observed region between the $\mathrm{La}_{2} \mathrm{O}_{2} \mathrm{CO}_{3}$ buffer layer and the PZT thin film.

$\mathrm{X}$-ray pole figure measurements are carried out to find the degree of orientation of the PZT thin films. The pole figure measurement of the $(110)$ orientation at $2 \theta=31^{\circ}$ gives a circle, which indicates that the films are (100) oriented out-of-plane with no specific in-plane texturing (see the inset of Figure 2). This is related to the good lattice match between $\mathrm{Ln}_{2} \mathrm{O}_{2} \mathrm{CO}_{3}$ and PZT on the one hand, and the absence of a driving force to orient the buffer layers in-plane, on the other hand. The (110) planes are aligned at an angle of $45^{\circ}$ to the silicon substrates, with a fwhm of $6^{\circ}$. The measurements on $\mathrm{La}, \mathrm{Nd}, \mathrm{Pr}$, and $\mathrm{Sm}$ based intermediate layers (thickness $\approx 10 \mathrm{~nm}$ ) produce very similar results. The experiments are repeated for different buffer layers based on $\mathrm{Sm}, \mathrm{Nd}$, Pr. They indicate the formation of $\mathrm{Sm}_{2} \mathrm{O}_{2} \mathrm{CO}_{3}(a=3.974 \AA, c=12.92 \AA), \mathrm{Nd}_{2} \mathrm{O}_{2} \mathrm{CO}_{3}(a=3.98 \AA$, $c=15.605 \AA)$, and $\operatorname{Pr}_{2} \mathrm{O}_{2} \mathrm{CO}_{3}(a=4.01 \AA, c=15.68 \AA)$ with a preferential out-of-plane orientation, and good lattice match (mismatch $<1 \%$ ) for all the buffer layers. 

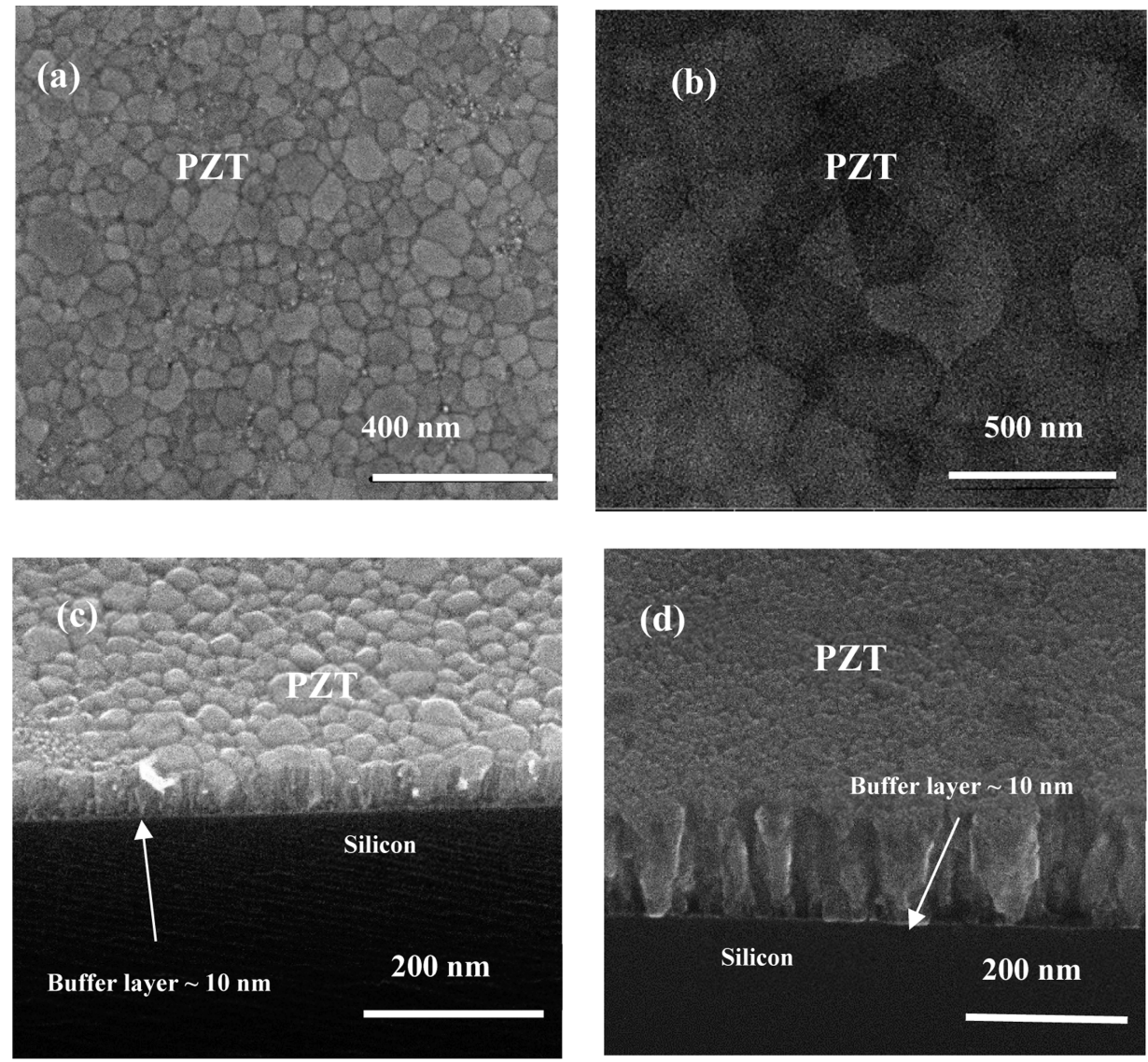

Figure 4. SEM top view of the PZT thin film (thickness $\sim 200 \mathrm{~nm}$, annealed at $630^{\circ} \mathrm{C}$ ) deposited on silicon substrate, coated with a (a) Nd-based intermediate layer (thickness $\approx 10 \mathrm{~nm}$, heat treated at $440{ }^{\circ} \mathrm{C}$ ). (b) La-based intermediate layer (thickness $\approx 10 \mathrm{~nm}$, heat treated at $500{ }^{\circ} \mathrm{C}$ ). Crosssection image of PZT thin film (thickness $\approx 200 \mathrm{~nm}$ ) (c) annealed at $630{ }^{\circ} \mathrm{C}(\mathrm{d})$ annealed at $560{ }^{\circ} \mathrm{C}$, on a silicon, coated with a La-based intermediate layer (thickness $\approx 10 \mathrm{~nm}$, heat treated at $500{ }^{\circ} \mathrm{C}$ ).

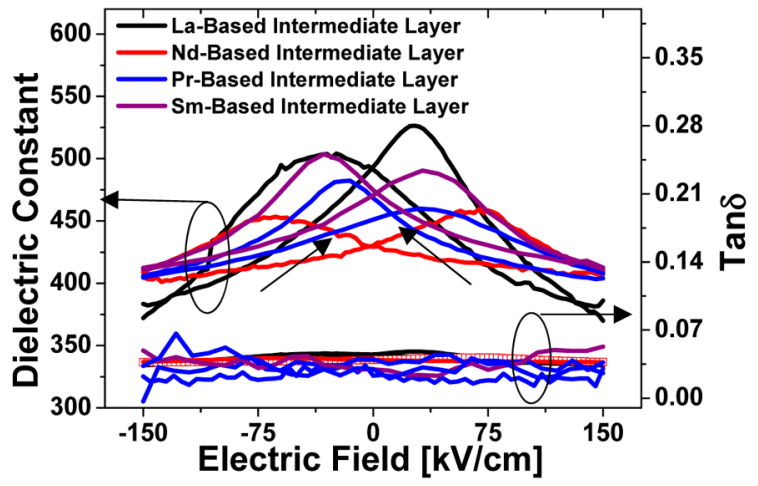

(a)

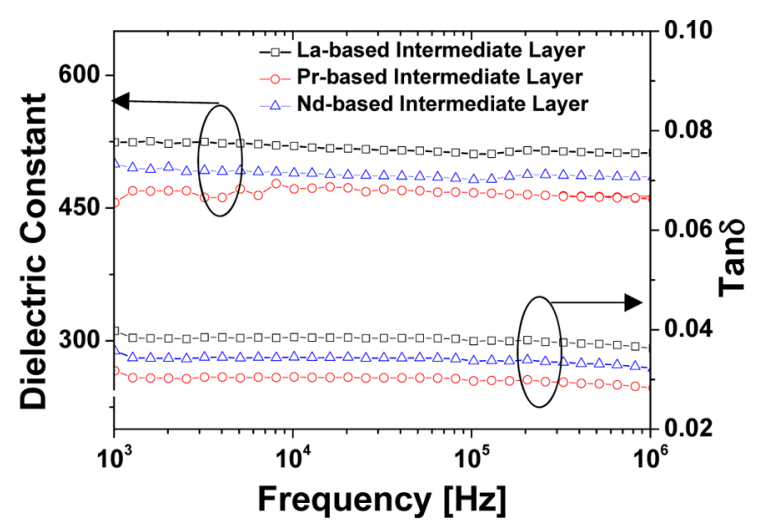

(b)

Figure 5. Small-signal ( $\mathrm{Vac}=0.1 \mathrm{~V}, f=10 \mathrm{kHz}$ ) relative dielectric constant of the PZT thin films (annealed at $630{ }^{\circ} \mathrm{C}$, thickness $\sim 600 \mathrm{~nm}$ ), on different template layers, (a) as a function of the DC bias voltage and $(\mathrm{b})$ as a function of frequency $\left(E_{\mathrm{dc}}=0 \mathrm{~V}\right)$.

Planar and cross sectional SEM images of representative PZT thin films deposited on $\mathrm{Si}$, on different template layers and annealed at different temperature are shown in Figure 4.

The surface morphology of the films confirms smooth, dense and uniform polygonal grains without any obvious secondary phase. The average grain sizes of the PZT films deposited on $\mathrm{Si}$, with intermediate template layers of $\mathrm{Nd}$ and $\mathrm{La}$ are estimated as $300 \mathrm{~nm}$, and $80 \mathrm{~nm}$, respectively. When the buffer layer thickness is below the critical thickness (buffer layer thickness $<5 \mathrm{~nm}$ ), the deposited PZT films show micro cracks and pin- holes on the film surface. The presence of the intercrystal voids and micro cracks increases the leakage current during the electrical measurements. These unwanted features are due to the thermal stress in the PZT thin films generated during multiple annealing steps, and the nonuniformities in the intermediate layers. The problem can be resolved by increasing the intermediate layer thickness. The cross sectional SEM images of the PZT shown as in Figure 4c, and Figure $4 \mathrm{~d}$ exhibits dense and columnar grains because of the heterogeneous nucleation growth. This growth behavior can also be 


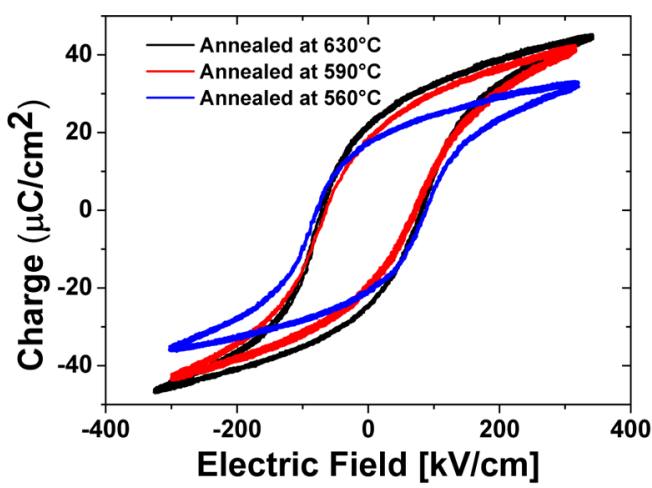

(a)

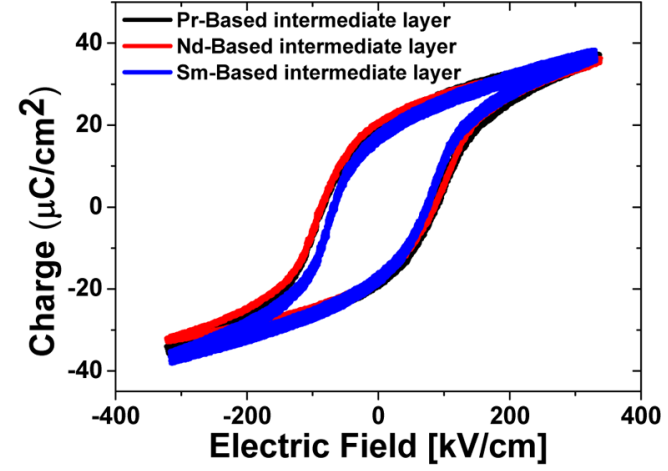

(b)

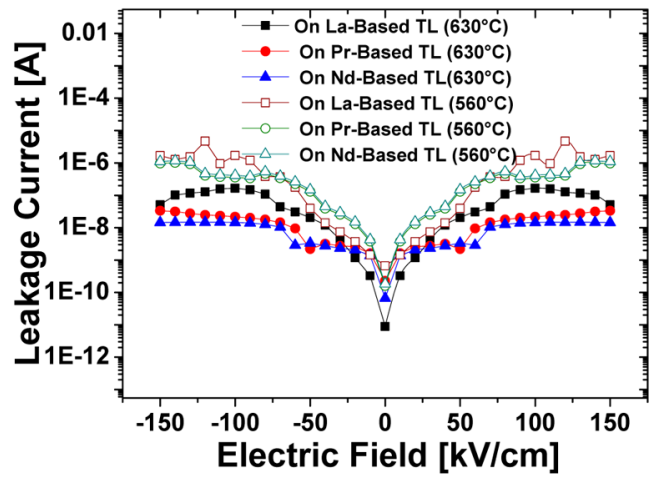

(c)

Figure 6. (a) $P-E$ hysteresis measurements of the PZT thin films (thickness $\sim 600 \mathrm{~nm}$, annealed at $630{ }^{\circ} \mathrm{C}$ ) (a) deposited on La-based intermediate layer annealed at different temperature (b) deposited on different buffer layers and annealed at $630^{\circ} \mathrm{C}$. The measurements are performed at $10 \mathrm{kHz}$. (c) Leakage current as a function of applied electric field for the PZT films deposited on different template layers, under different annealing treatments.

observed in the STEM imaging of a cross section (Figure 3). Micrometer-sized secondary phases like pyrochlore/fluorite phases are not observed in the SEM images. ${ }^{34}$ These observations are also confirmed by the lack of corresponding diffraction peaks and by the well-defined electrical measurements. Also the presence of the thin intermediate layer is evident from the SEM measurement, and the thickness matches with the data from ellipsometry measurements. Atomic force microscopy (AFM) measurements are carried out to estimate the rms roughness of the PZT films. A $5 \mu \mathrm{m}$ by $5 \mu \mathrm{m}$ square area is taken into consideration for measuring the average rms roughness value (see Supporting Information). The measured rms value for the roughness on La-, Nd-, Pr-, and Sm-based intermediate layers are $2.4,2.2,2.8$, and $2.6 \mathrm{~nm}$, respectively.

The capacitance-voltage $(C-V)$ hysteresis loops of the PZT thin films on different template layers are illustrated in Figure 5a. The $C-V$ measurement shows the small signal $(\mathrm{Vac}=0.1$ $\mathrm{V})$ capacitance as a function of the stepwise increased DC bias voltage. The measurements feature well-defined butterfly hysteresis loops that saturates at high electric fields with good dielectric tunability. Two maxima for the dielectric constants are observed depending on the direction of the electric field. The dielectric constant and the loss tangent extracted from the small signal analysis, in contrast to the large signal used in polarization-electric field (P-E) hysteresis measurements. We extract an effective dielectric constant from the capacitance which includes the effect of both the template and PZT layer. Two maxima for the dielectric constants are observed depending on the direction of the electric field. As seen from
Figure 5a, when the DC voltage increased from 0 to $200 \mathrm{kV} /$ $\mathrm{cm}$, the permittivity of the PZT films decreases from 540 to 360, 455 to 400, 502 to 413 , and 482 to 405 , for La-, Nd-, Pr-, and Sm-based template layers, respectively. The variations observed for the dielectric loss of the films is $0.045,0.02,0.04$, $0.36($ at $0 \mathrm{kV} / \mathrm{cm}$ ) and 0.038, 0.027, 0.02, 0.017 (at $200 \mathrm{kV} /$ $\mathrm{cm}$ ), respectively for PZT films on La-, Nd-, Pr-, and Sm-based template layers. Figure $5 \mathrm{~b}$ plots the permittivity and dielectric loss tangent as a function of frequency $(\mathrm{Vac}=0.1 \mathrm{~V})$ for the PZT thin films annealed at $630^{\circ} \mathrm{C}$, on $\mathrm{La}$, Pr, and $\mathrm{Nd}$ template layers. The measurements show a steady response of the dielectric constant and loss tangent up to $1 \mathrm{MHz}$.

Figure 6a represents the $P-E$ hysteresis measurements of the PZT thin films deposited on lanthanum based intermediate layers annealed at different temperatures. The measurements indicate well-saturated $P-E$ hysteresis loops, with a breakdown strength up to $800 \mathrm{kV} / \mathrm{cm}$. The remnant polarization (Pr), and coercive field (Ec) of the PZT films (thickness $-600 \mathrm{~nm}$ ) on a La-based template annealed at 630,590 , and $560{ }^{\circ} \mathrm{C}$ are 25,19 , and $19 \mu \mathrm{C} / \mathrm{cm}^{2}$ and 70,68 , and $75 \mathrm{kV} / \mathrm{cm}$, respectively. The results are comparable to strongly (100) textured epitaxial PZT thin films on $\mathrm{LSCO} / \mathrm{STO} / \mathrm{Si}$ substrate reported by Wang et.al, ${ }^{35}$ with $\operatorname{Pr}$ of $20 \mu \mathrm{C} / \mathrm{cm}^{2}$, however with a smaller coercive field of $40 \mathrm{kV} / \mathrm{cm}$. Figure $6 \mathrm{~b}$ shows hysteresis measurements of the PZT films annealed at $630{ }^{\circ} \mathrm{C}$ on different intermediate layers. The PZT films exhibit a coercive field of $89,78,85 \mathrm{kV} /$ $\mathrm{cm}$ and remnant polarization of 22,21 , and $20 \mu \mathrm{C} / \mathrm{cm}^{2}$, on Nd-, Sm-, and Pr-based intermediate layers, respectively. The leakage current with applied electric field in Figure $6 \mathrm{c}$ is in the 


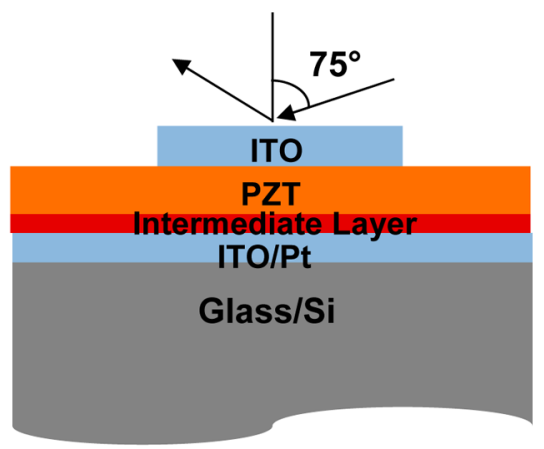

(a)

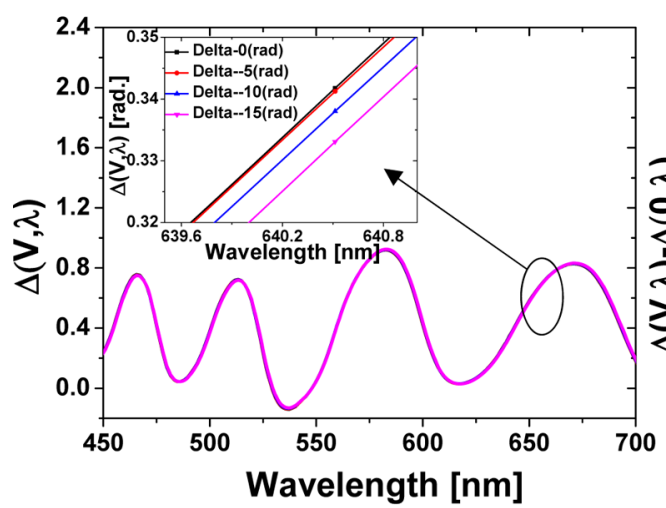

(b)

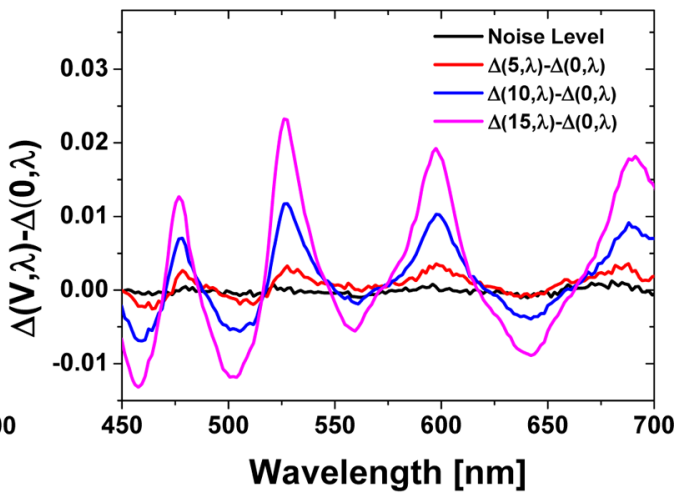

(c)

Figure 7. (a) Schematic representation of the sample stack and the incident light beams from the ellipsometer. (b) Observed variation in $\Delta$, as a function of the applied voltage. (c) Change observed in $\Delta(V, \lambda)-\Delta(0, \lambda)$ at $0 \mathrm{~V}$ (Noise level) 5,10 , and $15 \mathrm{~V}$.

order of $0.1 \mu \mathrm{A}$ at $150 \mathrm{kV} / \mathrm{cm}$ for the PZT films annealed at $630{ }^{\circ} \mathrm{C}$ on different buffer layers. However, as the annealing temperature is decreased to $560{ }^{\circ} \mathrm{C}$, leakage current increases by an order of magnitude. The increase in the leakage current corresponds well with the decrease in the grain sizes seen in the SEM measurements for the samples annealed at lower temperatures.

Electro-optic Characterizations. A spectroscopic ellipsometry based approach has been used to estimate the electric field induced birefringence, similar to the method described in previous reports. ${ }^{36,37}$ With this method it is possible to extract an effective electro-optic (EO) coefficient without the need to do the full fitting of the layer stack parameters. PZT films of thickness $800 \mathrm{~nm}$ have been deposited on either ITO/glass substrates or $\mathrm{Pt} / \mathrm{Si}$ substrates for the EO measurements. To facilitate the ellipsometry measurements, a top transparent conductive ITO thin film of thickness $30 \mathrm{~nm}$ is deposited, by ebeam evaporation and subsequent heat treatment at $300{ }^{\circ} \mathrm{C}$ to obtain sufficient conductivity and transparency of the ITO. The PZT films are structurally characterized prior to the electrooptic measurements. The X-ray diffraction measurements show strong preferential orientation along the (100) crystallographic direction with sharp diffraction peaks corresponding to the tetragonal PZT (52/48). The SEM measurements show well packed polygonal crystal grains, with an rms roughness of 3.1 $\mathrm{nm}$ as determined by AFM. To confine the light beam completely within the electrode, the top electrode is patterned to a size of $1.5 \mathrm{~cm} \times 0.5 \mathrm{~cm}$ using a metal shadow mask. The schematic representation of a typical sample layer stacks used for the electro-optic measurements are shown in Figure 7a. A DC voltage is applied between the top and bottom contact to introduce a change in the refractive index inside the PZT film. Note that, we use a relatively large electrode size, but none of the good quality PZT films exhibited a breakdown for field strength up to $200 \mathrm{kV} / \mathrm{cm}$. However, in our measurement the films are of such quality that the leakage current is on the order of $10^{-7} \mathrm{~A}$ which allows the prolonged electro-optic measurements without any dielectric breakdown (see the Supporting Information). The light is incident under an angle of $75^{\circ}$ onto the ITO $(30 \mathrm{~nm}) / \mathrm{PZT}(800 \mathrm{~nm}) / \mathrm{La}_{2} \mathrm{O}_{2} \mathrm{CO}_{3}(10 \mathrm{~nm}) / \mathrm{ITO} /$ Glass sample for the ellipsometry measurements. The measurement gives a value for the ellipsometric angles $\psi$ and $\Delta$, which are used to define the complex reflectance ratio of the P- to the S-polarized light.

$$
r_{\mathrm{p}} / r_{\mathrm{s}}=\tan (\psi) \times \exp (i \Delta)
$$

where the $\tan \psi$ is the amplitude reflectance ratio and $\Delta$ is the phase shift between the p- and s-polarized light after reflection. In the conventional procedure for analyzing the ellipsometry data, the dielectric function are numerically optimized for each of the layers assuming a certain dispersion relation. ${ }^{36}$ The dielectric function of each layer arises from a fitting procedure considering the transmission, reflection, and absorption of the light on all possible interfaces. However, each layer needs to be modeled very precisely to extract the small variations introduced by the application of electric fields. The optimization of the model is carried out until the residual mean square is converged to the minimum. However, this method is very tedious in our sample structures as at least 10 parameters ( $n$ and $k$ for each of the 5 layers) need to be optimized simultaneously. 
To model the small variations in the refractive index, induced by an applied electric field, we followed a method of differentiating the phase reflectance ratio $(\Delta)$ obtained from the ellipsometry measurements. We assume that the variations of the ellipsomtery $\Delta$ angles are only the result of the changes in the refractive index inside the PZT film, and that absorption does not play a role in modeling the $\Delta$ angles. According to previous reports the contribution to the phase change by the thickness variation in PZT film because of the piezoelectric effect is negligible compared to that of the refractive index change. ${ }^{38}$ As the variation expected in the refractive index is in the order of $10^{-3}$, it is assumed that the reflections from the different layer boundaries have not introduced any phase variations in the $\Delta$ angles with applied voltages.

Figure 7a represents the ellipsometry $\Delta$ angles measured for the ITO/PZT $/ \mathrm{La}_{2} \mathrm{O}_{2} \mathrm{CO}_{3} / \mathrm{ITO} /$ glass sample. The value of $\Delta$ at different applied electric fields indicates clear variation with applied voltage. The close up of the pattern observed in the range of $639-641 \mathrm{~nm}$ clearly demonstrates the changes observed in $\Delta$. The $\Delta$ angle at a given electric field $\Delta(V, \lambda)$ shows a clear shift with the applied electric field, with reference to the value for $\Delta$ at $0 \mathrm{~V}(\Delta(0, \lambda))$, clearly indicating the linear EO Pockels effect from the film. The variation observed in the $\Delta$ angle at three different voltages are shown in Figure $7 \mathrm{c}$.

The background noise in the measurements correspond to the uncertainty in the experimental measurement of the ellipsometry angles, which may originate from fluctuations in the intensity of incident light over time. The influence of the noise on the $\Delta$ spectrum is estimated by mutually subtracting two $\Delta$ spectrum at the same voltage (see Supporting Information). It is clear that the changes due to voltage are well above the noise level in the $\Delta$ spectrum, which confirms the electric field introduced phase change.

The method consists of estimating the effective wavelength change in PZT because of the change in the refractive index. To evaluate this, we differentiate the $\Delta$ angle, assuming that all other parameters except the optical permittivity tensor of (PZT) remain the same. We obtained small changes in the effective wavelength caused by the change refractive index $n(\mathrm{PZT})$, corresponding to small variation in the applied voltage using the equation

$$
\Delta \lambda=\left[\frac{\frac{\partial \Delta(V, \lambda)}{\partial V}}{\frac{\partial \Delta(0, \lambda)}{\partial \lambda}}\right] \times \mathrm{d} V
$$

The $(\partial \Delta(0, \lambda) / \partial \lambda)$ spectrum is obtained from the $\Delta(0, \lambda)$ spectrum (see the Supporting Information), by calculating $\Delta(0, \lambda)-\Delta(0, \lambda+0.006 \mu \mathrm{m})$. It is noted that the spectrum resembles the $(\partial \Delta(V, \lambda) / \partial \lambda)$ spectrum except for the different amplitude. Since a wavelength shift of $0.006 \mu \mathrm{m}$ corresponds to the oscillatory spectrum in $(\partial \Delta(0, \lambda) / \partial \lambda)$, by comparison we can extract the effective wavelength shift in the actual oscillatory function given in Figure $7 c$. The $(\partial \Delta(0, \lambda) / \partial \lambda),(\partial \Delta(V, \lambda) / \partial \lambda)$ and the noise level spetrums are plotted in (see the Supporting Information Figure $\mathrm{S} 3 \mathrm{c}-\mathrm{e}$ ). Since the effective wavelength of the light propagating in a medium of refractive index $\mathrm{n}$ is given by $\lambda / n$, the change in the wavelength observed is converted to corresponding $\Delta n$ through $\Delta n=(n / \lambda) \Delta \lambda$.Finally, the effective pockels coefficients are determined from $\Delta n$, using

$$
\Delta n=\frac{1}{2} \times n^{3} \times r_{\text {eff }} \times E
$$

Figure $8 \mathrm{a}$ and $\mathrm{b}$ represents the electric field refractive index change and the corresponding effective pockels coefficient of

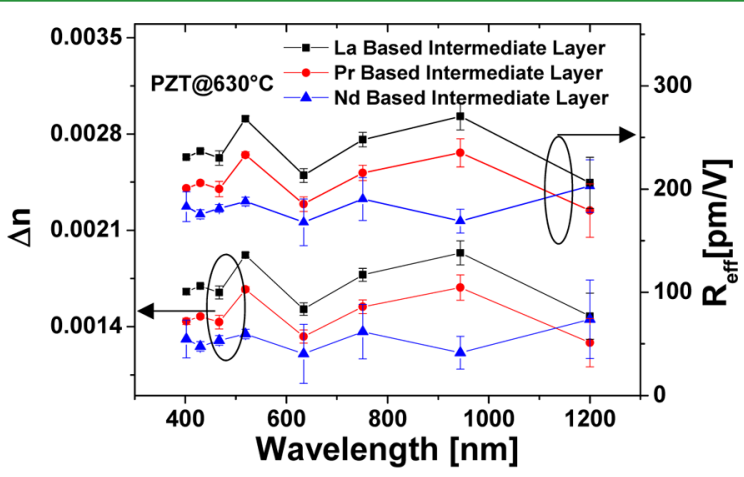

(a)

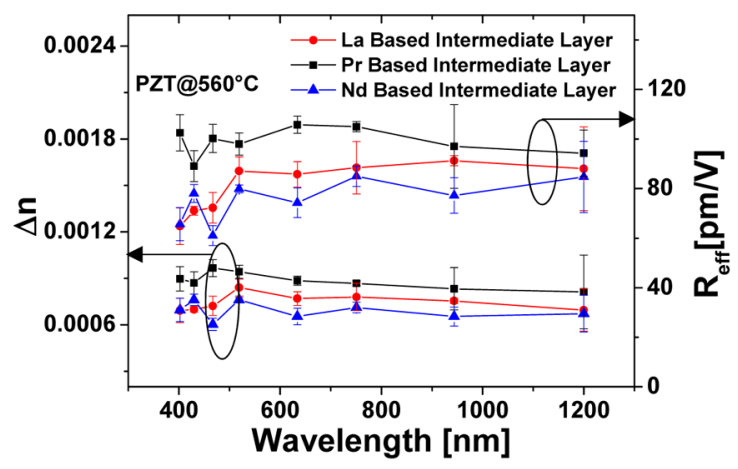

(b)

Figure 8. Refractive index change and the effective electro optic coefficients of the PZT thin film (thickness $\sim 800 \mathrm{~nm}$ ) annealed at (a) 630 and (b) $560{ }^{\circ} \mathrm{C}$, on different lanthanide based template layers.

the PZT thin films deposited on different lanthanide based template layers with $10 \mathrm{~nm}$ thickness annealed at different temperature. The films possess strong effective EO coefficients. The graph indicates which annealing temperature and buffer layer properties result in the highest EO coefficient. The PZT films annealed at $630{ }^{\circ} \mathrm{C}$ on La, Pr and $\mathrm{Nd}$ buffer layers exhibit a linear pockels coefficient of 240, 215, and $200 \mathrm{pm} / \mathrm{V}$, respectively, at the wavelength of $630 \mathrm{~nm}$. Even though a significant change in the pockels effects is observed by reducing the annealing temperature to $560{ }^{\circ} \mathrm{C}$, the electro-optic effects of the films are still comparable to previously reported results with the films annealed above $600{ }^{\circ} \mathrm{C} .{ }^{39}$ The pockels coefficient of the films annealed at $560{ }^{\circ} \mathrm{C}$ are reduced to $115 \mathrm{pm} / \mathrm{V}, 96 \mathrm{pm} /$ $\mathrm{V}$ and $89 \mathrm{pm} / \mathrm{V}$ for the films deposited on La, $\mathrm{Pr}$ and $\mathrm{Nd}$ intermediate layers. The effective linear electro-optic coefficients $\left(r_{\text {eff }}\right)$ measured here are comparable to the previously reported values. Epitaxially grown (100) oriented PZT (52/48) thin films on a Nd:STO (100) substrate show an $r_{\text {eff }}$ of 165 $\mathrm{pm} / \mathrm{V}^{36}$ Masuda et.al ${ }^{40}$ has demonstrated further improvement in $r_{\text {eff }}$ up to $498 \mathrm{pm} / \mathrm{V}$ for epitaxial (111) oriented PZT (52/ 48) thin film on Nd.STO (111) substrate by controlling the crystal phases in the films with a modified annealing procedure and an electro-optic modulator of $40 \mathrm{GHz}$ speed is also demonstrated with this method. ${ }^{41}$ Even though a strong electro-optic properties of the PZT thin films are demonstrated on different substrates, only few reports are on silicon. ${ }^{42}$ Stateof-art PZT/Si photonic devices use the piezo-electric properties of the film, to break the Si crystal symmetry and to introduce the birefringence in the $\mathrm{Si}^{43,44}$ However, the change in the 
refractive index observed in these devices are an order of magnitude smaller compared to the electro-optics effects measured here.

\section{CONCLUSION}

In conclusion, a novel strategy for fabricating highly textured PZT thin films on silicon has been developed, by utilizing a chemical solution deposition route. We have developed a number of novel ultrathin lanthanide based intermediate layers, as template layers for textured thin film growth on different substrates. The structural characterizations of the films demonstrate strong preferential thin film growth, with well packed, crack free polygonal crystal grains. The PZT thin films have a strong columnar structure with a high degree of orientation $(>99.8 \%)$ regardless of the thin film growth conditions. The dielectric constant measurement of the films shows the expected trend with a large dielectric constant $\sim 600$ (scaled by the buffer layer thickness, dielectric constant, and electrode parameters). The hysteresis and leakage current measurement shows low coercive field, strong remnant polarization, and large breakdown voltage. Strong electrooptic properties of the as-deposited thin films (ranging from 120 to $240 \mathrm{pm} / \mathrm{V}$ ), estimated by the ellipsomtery technique, indicate that this PZT film is an ideal candidate for silicon nanophotonics EO modulators. The novel deposition method offers a low cost and simple method to produce good quality, strongly electro-optic PZT thin films on crystalline and amorphous $\left(\mathrm{SiO}_{2}\right.$ and $\left.\mathrm{Si}_{3} \mathrm{~N}_{4}\right)$ substrates for a variety of integrated photonics and electronics applications, where the properties of the films can be tuned for applications spanning from microwave to the optical frequencies.

\section{ASSOCIATED CONTENT}

\section{S Supporting Information}

Some details of the X-ray diffraction measurements, pole figures, atomic force microscopy (AFM) measurements and the details of the electro-optic characterization of the PZT films. The Supporting Information is available free of charge on the ACS Publications website at DOI: 10.1021/acsami.5b01781.

\section{AUTHOR INFORMATION}

\section{Corresponding Author}

*E-mail: John.PuthenParampilGeorge@elis.Ugent.be.

\section{Notes}

The authors declare no competing financial interest.

\section{ACKNOWLEDGMENTS}

This research was supported by the Interuniversity Attraction Poles program of the Belgian Science Policy Office, under grant IAP P7-35 (Photonics@be)

\section{REFERENCES}

(1) Kobayashi, T.; Oyama, S.; Takahashi, M.; Maeda, R.; Itoh, T. Microelectromechanical Systems-Based Electrostatic Field Sensor Using $\mathrm{Pb}(\mathrm{Zr}, \mathrm{Ti}) \mathrm{O}_{3}$ Thin Films. Jpn. J. Appl. Phys. 2008, 47, 7533.

(2) Xiao, B.; Avrutin, V.; Liu, H. Y.; Ozgur, U.; Morkoc, H.; Lu, C. Z. Large Pyroelectric Effect in Undoped Epitaxial $\mathrm{Pb}(\mathrm{Zr}, \mathrm{Ti}) \mathrm{O}_{3}$ Thin Films on $\mathrm{SrTiO}_{3}$ Substrates. Appl. Phys. Lett. 2008, 93, No. 052913.

(3) Lei, X. Y.; Remiens, D.; Sama, N.; Chen, Y.; Mao, C. L.; Dong, X. L.; Wang, G. S. Dielectric, Ferroelectric and Piezoelectric Properties of 100-Oriented $\mathrm{Pb}_{0.4} \mathrm{Sr}_{0.6} \mathrm{TiO}_{3}$ Thin Film Sputtered on $\mathrm{LaNiO}_{3}$ Electrode. J. Cryst. Growth 2012, 347, 15.
(4) Zhu, M. M.; Du, Z. H.; Ma, J. Influence of Crystal Phase and Transparent Substrates on Electro-Optic Properties of Lead Zirconate Titanate Films. J. Appl. Phys. 2010, 108, No. 113119.

(5) Eom, C. B.; Trolier-McKinstry, S. Thin-Film Piezoelectric MEMS. MRS Bull. 2012, 37, 1007-1021.

(6) Sriram, S.; Bhaskaran, M.; Holland, A. S.; Short, K. T.; Latella, B. A. Measurement of High Piezoelectric Response of Strontium-Doped Lead Zirconate Titanate Thin Films Using a Nanoindenter. J. Appl. Phys. 2007, 101, No. 104910.

(7) Bin, C.; Zhenghu, Z.; Yiwei, L.; Qing-Feng, Z.; Yali, X.; Huali, Y.; Guohong, D.; Zhixiang, L.; Gaojie, X.; Run-Wei, L. Tunable Photovoltaic Effects in Transparent $\mathrm{Pb}\left(\mathrm{Zr}{ }_{0.53}, \mathrm{Ti}_{0.47}\right) \mathrm{O}_{3}$ Capacitors. Appl. Phys. Lett. 2012, 100, No. 173903.

(8) Luo, C.; Cao, G. Z.; Shen, I. Y. Development of a LeadZirconate-Titanate (PZT) Thin-Film Microactuator Probe for Intracochlear Applications. Sens. Actuators, A 2013, 201, 1-9.

(9) Thapliya, R.; Okano, Y.; Nakamura, S. Electrooptic Characteristics of Thin-Film PLZT Waveguide Using Ridge-Type MachZehnder Modulator. J. Lightwave Technol. 2003, 21, 1820-1827.

(10) Xiong, C.; Pernice, W. H. P.; Ngai, J. H.; Reiner, J. W.; Kumah, D.; Walker, F. J.; Ahn, C. H.; Tang, H. X. Active Silicon Integrated Nanophotonics: Ferroelectric $\mathrm{BaTiO}_{3}$ Devices. Nano Lett. 2014, 14, 1419-1425.

(11) Kim, J.; Yang, S. A.; Choi, Y. C.; Han, J. K.; Jeong, K. O.; Yun, Y. J.; Kim, D. J.; Yang, S. M.; Yoon, D.; Cheong, H.; Chang, K. S.; Noh, T. W.; Bu, S. D. Ferroelectricity in Highly Ordered Arrays of UltraThin-Walled $\mathrm{Pb}(\mathrm{Zr}, \mathrm{Ti}) \mathrm{O}_{3}$ Nanotubes Composed of Nanometer-Sized Perovskite Crystallites. Nano Lett. 2008, 8, 1813-1818.

(12) Datta, A.; Mukherjee, D.; Witanachchi, S.; Mukherjee, P. Hierarchically Ordered Nano-Heterostructured PZT Thin Films with Enhanced Ferroelectric Properties. Adv. Funct. Mater. 2014, 24, 26382647.

(13) Chen, X.; Xu, S. Y.; Yao, N.; Shi, Y. 1.6 V Nanogenerator for Mechanical Energy Harvesting Using PZT Nanofibers. Nano Lett. 2010, 10, 2133-2137.

(14) Datta, A.; Mukherjee, D.; Hordagoda, M.; Witanachchi, S.; Mukherjee, P.; Kashid, R. V.; More, M. A.; Joag, D. S.; Chavan, P. G. Controlled $\mathrm{Ti}$ Seed Layer Assisted Growth and Field Emission Properties of $\mathrm{Pb}\left(\mathrm{Zr}_{0.52} \mathrm{Ti}_{0.48}\right) \mathrm{O}_{3}$ Nanowire Arrays. ACS Appl. Mater. Interfaces 2013, 5, 6261-6267.

(15) Tang, P. S.; Towner, D. J.; Hamano, T.; Meier, A. L.; Wessels, B. W. Electrooptic Modulation up to $40 \mathrm{GHz}$ in a Barium Titanate Thin Film Waveguide Modulator. Opt. Express 2004, 12, 5962-5967.

(16) Wooten, E. L.; Kissa, K. M.; Yi-Yan, A.; Murphy, E. J.; Lafaw, D. A.; Hallemeier, P. F.; Maack, D.; Attanasio, D. V.; Fritz, D. J.; McBrien, G. J.; Bossi, D. E. A Review of Lithium Niobate Modulators for FiberOptic Communications Systems. IEEE J. Sel. Top. Quantum Electron. 2000, 6, 69-82.

(17) Rabiei, P.; Ma, J. C.; Khan, S.; Chiles, J.; Fathpour, S. Heterogeneous Lithium Niobate Photonics on Silicon Substrates. Opt. Express 2013, 21, 25573-25581.

(18) Masuda, S.; Seki, A.; Shiota, K.; Masuda, Y. Mach-Zehnder Interferometer-Type Photonic Switches Based on Epitaxially Grown Lanthanum-Modified Lead Zirconate Titanate Films. J. Lightwave Technol. 2011, 29, 209-214.

(19) Abel, S.; Stoeferle, T.; Marchiori, C.; Rossel, C.; Rossell, M. D.; Erni, R.; Caimi, D.; Sousa, M.; Chelnokov, A.; Offrein, B. J.; Fompeyrine, J. A Strong Electro-Optically Active Lead-Free Ferroelectric Integrated on Silicon. Nat. Commun. 2014, 4, 1-6.

(20) Cooney, T. G.; Francis, L. F. Processing of Sol-Gel Derived PZT Coatings on Non-Planar Substrates. J. Micromech. Microeng. 1996, 6, 291-300.

(21) Frunza, R.; Ricinschi, D.; Gheorghiu, F.; Apetrei, R.; Luca, D.; Mitoseriu, L.; Okuyama, M. Preparation and Characterisation of PZT Films by RF-Magnetron Sputtering. J. Alloy. Compd. 2011, 509, 62426246.

(22) Menou, N.; Funakubo, H. (111)-Oriented $\mathrm{Pb}(\mathrm{Zr}, \mathrm{Ti}) \mathrm{O}_{3}$ Films Deposited on $\mathrm{SrRuO}_{3} / \mathrm{Pt}$ Electrodes: Reproducible Preparation by 
Metal Organic Chemical Vapor Deposition, Top Electrode Influence, and Reliability. J. Appl. Phys. 2007, 102, No. 114105.

(23) Borowiak, A. S.; Niu, G.; Pillard, V.; Agnus, G.; Lecoeur, P.; Albertini, D.; Baboux, N.; Gautier, B.; Vilquin, B. Pulsed Laser Deposition of Epitaxial Ferroelectric $\mathrm{Pb}(\mathrm{Zr}, \mathrm{Ti}) \mathrm{O}_{3}$ Films on Silicon Substrates. Thin Solid Films 2012, 520, 4604-4607.

(24) Lin, Y.; Zhao, B. R.; Peng, H. B.; Xu, B.; Chen, H.; Wu, F.; Tao, H. J.; Zhao, Z. X.; Chen, J. S. Growth and Polarization Features of Highly (100) Oriented $\mathrm{Pb}\left(\mathrm{Zr}_{0.53} \mathrm{Ti}_{0.47}\right) \mathrm{O}_{3}$ Films on Si with Ultrathin $\mathrm{SiO}_{2}$ Buffer Layer. Appl. Phys. Lett. 1998, 73, 2781-2783.

(25) Chen, L.; Shen, M. R.; Fang, L.; Xu, Y. Microstructure Control of $(\mathrm{Pb}, \mathrm{Sr}) \mathrm{TiO}_{3}$ Films on $\mathrm{Pt} / \mathrm{Ti} / \mathrm{SiO}_{2} / \mathrm{Si}$ Substrates by a $\mathrm{TiO}_{2}$ Buffer Layer. Thin Solid Films 2008, 516, 1285-1289.

(26) Yu, J.; Meng, X. J.; Sun, J. L.; Huang, Z. M.; Chu, J. H. Optical and Electrical Properties of Highly (100)-Oriented $\mathrm{PbZr}_{1-x} \mathrm{Ti}_{x} \mathrm{O}_{3}$ Thin Films on the $\mathrm{LaNiO}_{3}$ Buffer Layer. J. Appl. Phys. 2004, 96, 2792-2799.

(27) Shih, W. C.; Yen, Z. Z.; Liang, Y. S. Preparation of Highly cAxis-Oriented PZT Films on Si Substrate with Mgo Buffer Layer by the Sol-Gel Method. J. Phys. Chem. Solids 2008, 69, 593-596.

(28) Chen, L.; Reano, R. M. Compact Electric Field Sensors Based on Indirect Bonding of Lithium Niobate to Silicon Microrings. Opt. Express 2012, 20, 4032-4038.

(29) Kim, D. M.; Eom, C. B.; Nagarajan, V.; Ouyang, J.; Ramesh, R.; Vaithyanathan, V.; Schlom, D. G. Thickness Dependence of Structural and Piezoelectric Properties of Epitaxial $\mathrm{Pb}\left(\mathrm{Zr}_{0.52} \mathrm{Ti}_{0.48}\right) \mathrm{O}_{3}$ Films on $\mathrm{Si}$ and $\mathrm{SrTiO}_{3}$ Substrates. Appl. Phys. Lett. 2006, 88, No. 142904.

(30) Balboul, B. A. A.; Myhoub, A. Y. Z. The Characterization of the Formation Course of Neodymium Oxide from Different Precursors: A Study of Thermal Decomposition and Combustion Processes. J. Anal. Appl. Pyrolysis 2010, 89, 95-101.

(31) Klingenberg, B.; Vannice, M. A. Influence of Pretreatment on Lanthanum Nitrate, Carbonate, and Oxide Powders. Chem. Mater. 1996, 8, 2755-2768.

(32) Shirsat, A. N.; Ali, M.; Kaimal, K. N. G.; Bharadwaj, S. R.; Das, D. Thermochemistry of $\mathrm{La}_{2} \mathrm{O}_{2} \mathrm{CO}_{3}$ Decomposition. Thermochim. Acta 2003, 399, 167-170.

(33) Olafsen, A.; Fjellvag, H. Synthesis of Rare Earth Oxide Carbonates and Thermal Stability of $\mathrm{Nd}_{2} \mathrm{O}_{2} \mathrm{CO}_{3}$ ii. J. Mater. Chem. 1999, 9, 2697-2702.

(34) Lefevre, M. J.; Speck, J. S.; Schwartz, R. W.; Dimos, D.; Lockwood, S. J. Microstructural Development in Sol-Gel Derived Lead Zirconate Titanate Thin Films: The Role of Precursor Stoichiometry and Processing Environment. J. Mater. Res. 1996, 11, 2076-2084.

(35) Wang, Y.; Ganpule, C.; Liu, B. T.; Li, H.; Mori, K.; Hill, B.; Wuttig, M.; Ramesh, R.; Finder, J.; Yu, Z.; Droopad, R.; Eisenbeiser, K. Epitaxial Ferroelectric $\mathrm{Pb}(\mathrm{Zr}, \mathrm{Ti}) \mathrm{O}_{3}$ Thin Films on $\mathrm{Si}$ Using $\mathrm{SrTiO} 3$ Template Layers. Appl. Phys. Lett. 2002, 80, 97-99.

(36) Kang, T. D.; Xiao, B.; Avrutin, V.; Ozgur, U.; Morkoc, H.; Park, J. W.; Lee, H. S.; Lee, H.; Wang, X. Y.; Smith, D. J. Large Electro-Optic Effect in Single-Crystal $\mathrm{Pb}(\mathrm{Zr}, \mathrm{Ti}) \mathrm{O}_{3}(001)$ Measured by Spectroscopic Ellipsometry. J. Appl. Phys. 2008, 104, No. 093103.

(37) Akazawa, H.; Shimada, M. Spectroellipsometric Approach to Determine Linear Electro-Optic Coefficient of C-Axis-Oriented LiNbO3 Thin Films. J. Appl. Phys. 2005, 98, No. 113501.

(38) Spirin, V. V.; Lee, C. H.; No, K. S. Measurement of the Pockels Coefficient of Lead Zirconate Titanate Thin Films by a Two-Beam Polarization Interferometer with a Reflection Configuration. J. Opt. Soc. Am. B 1998, 15, 1940-1946.

(39) Lee, C.; Spirin, V.; Song, H.; No, K. Drying Temperature Effects on Microstructure, Electrical Properties and Electro-Optic Coefficients of Sol-Gel Derived PZT Thin Films. Thin Solid Films 1999, 340, 242-249.

(40) Masuda, S.; Seki, A.; Masuda, Y. Influence of Crystal Phases on Electro-Optic Properties of Epitaxially Grown Lanthanum-Modified Lead Zirconate Titanate Films. Appl. Phys. Lett. 2010, 96, No. 072901. (41) Masuda, S.; Seki, A.; Shiota, K.; Hara, H.; Masuda, Y. ElectroOptic and Dielectric Characterization of Ferroelectric Films for High-
Speed Optical Waveguide Modulators. J. Appl. Phys. 2011, 109, No. 124108.

(42) Kurihara, K.; Kondo, M.; Sato, K.; Ishii, M.; Wakiya, N.; Shinozaki, K. Electrooptic Properties of Epitaxial Lead Zirconate Titanate Films on Silicon Substrates. Jpn. J. Appl. Phys., Part 1 2007, 46, 6929-6932.

(43) Sebbag, Y.; Goykhman, I.; Desiatov, B.; Nachmias, T.; Yoshaei, O.; Kabla, M.; Meltzer, S. E.; Levy, U. Bistability in Silicon Microring Resonator Based on Strain Induced by a Piezoelectric Lead Zirconate Titanate Thin Film. Appl. Phys. Lett. 2012, 100, No. 141107.

(44) Tsia, K. K.; Fathpour, S.; Jalalia, B. Electrical Tuning of Birefringence in Silicon Waveguides. Appl. Phys. Lett. 2008, 92, No. 061109. 